\title{
Access Signaling and IN-based Mobility Management for Wireless ATM Systems
}

\author{
Nikos H. Loukas*, Christos K. Xenakis ${ }^{*}$, Lazaros Merakos* and Iakovos Venieris ${ }^{+}$ \\ *University of Athens, Department of Informatics, Communication Networks Laboratory \\ (loukas | xenakis | merakos @ di.uoa.gr) \\ ${ }^{+}$National Technical University of Athens, Electrical and Computer Engineering Dept. \\ (ivenieri@cc.ece.ntua.gr)
}

\begin{abstract}
This paper focuses on the design of signaling and mobility control protocols for Wireless ATM (W-ATM) systems based on the Intelligent Network (IN) concepts. The employed signaling protocol architecture aims for private W-ATM networks to access the core BISDN/ATM infrastructure. The proposed design intents to minimize the changes required to the wired ATM network signaling to accommodate mobility features, by taking advantage of the well-developed capability sets. A signaling performance evaluation is carried-out to demonstrate the impact of the proposed signaling architecture onto various performance measures and to identify the behavior of the system under various signaling loads in order to reveal the limits of the signaling system stability.
\end{abstract}

\section{INTRODUCTION}

The development needs of a Wireless ATM (W-ATM) access network, capable of offering broadband services and mobility features to the wireless user, imposes a number of important considerations on the design of signaling protocols. The main challenge is to consolidate the wireless and wired ATM technology into a seamless network infrastructure, capable of providing qualitatively similar service attributes to the mobile user as in fixed ATM. To this end, this paper elaborates on the design of W-ATM signaling and control protocols, deployed as extensions to the standard signaling capabilities to cater for mobility.

The proposed signaling architecture is based on the design principles of fixed broadband access networks and on the Intelligent Network (IN) concepts for the support of terminal mobility. The resulting protocol model aims for private W-ATM systems to access the core B-ISDN infrastructure, but they can be easily adapted to fulfill the access signaling requirements of public environment wireless ATM system for future Personal Communication Systems (PCS). The design carried out in this paper is quantitatively studied by simulation. The obtained results capture the effect of the proposed signaling structure on the performance of call and mobility control in terms of call set-up and handover mean processing delay and buffering requirements at the system's signaling processors, and can be used for network design purposes in a large scale, private environment supporting many users.

The rest of this paper is organized as follows. Section 2 introduces the system's architecture and presents the protocol stacks. Section 3 describes the signaling and control procedures encountered in the mobile system under study and gives the corresponding information flows. Section 4 presents the simulation model used to evaluate the proposed architecture and the obtained numerical results. Finally, Section 5 contains our conclusions.

\section{SYSTEM ARCHITECTURE}

\section{A. Network Architecture}

The network configuration considered here assumes a number of local-area, W-ATM access systems, interconnected as shown Fig. 1, providing continuous radio coverage within a large-scale, business environment.

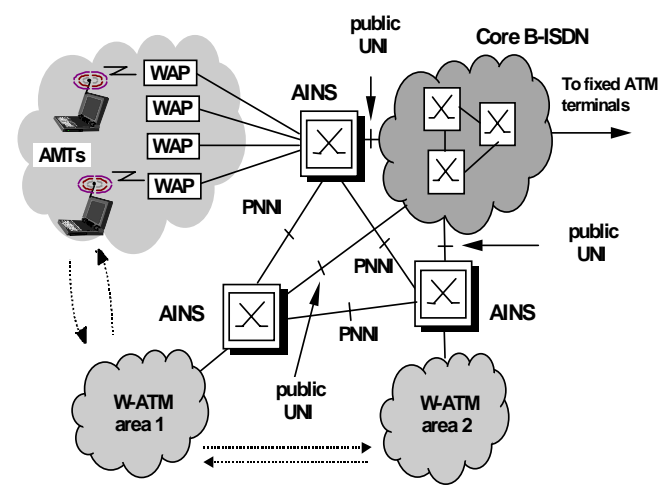

Figure 1 - High-Level Network Architecture

Each W-ATM area is formed by an ATM/IN Switch (AINS) and its attached Wireless Access Points (WAPs). AINS incorporates a standard ATM switch, and it is the focal network element providing the basic call and bearer control, the switching and the transmission functions, as well as the additional IN service and control logic required to support mobility. AINS realizes the interworking functions required between the wireless access network and the fixed B-ISDN.

The Wireless Access Points (WAPs) include the radio link management functions and serve the ATM Mobile Terminals (AMTs) in their coverage area through a shared radio channel. WAPs act as gateways with no switching capability for communication between mobile terminals and the fixed ATM. The WAPs extract the encapsulated ATM cells from the Medium Access Control (MAC) frames and forward them to the fixed ATM network through ATM Virtual Connections (VCs). The AMTs are considered as the equipment of the mobile end-user, and contain the wireless ATM radio adapted cards interfacing the air interface.

\section{B. Protocol Stacks}


The Control-plane protocol stacks are given in Fig. 2. The functional entities sitting on the top of the signaling protocol layers are based on a generic IN functional model to support mobility, [1]. The Call Control (CC) signaling is realized between the Mobile Call Control Function (MCCF) at the AMT and the Call Control Function (CCF) at the AINS. This includes an extended ATM UserNetwork-Interface (UNI) signaling protocol, [2], (denoted as Q.2931*), for the set-up, modification and release of calls, over the Signaling ATM Adaptation Layer (S-AAL) and the ATM layer protocols. The major enhancements required in the current signaling standards are related to the support of the handover function (e.g., inclusion of handover-specific messages), [3].

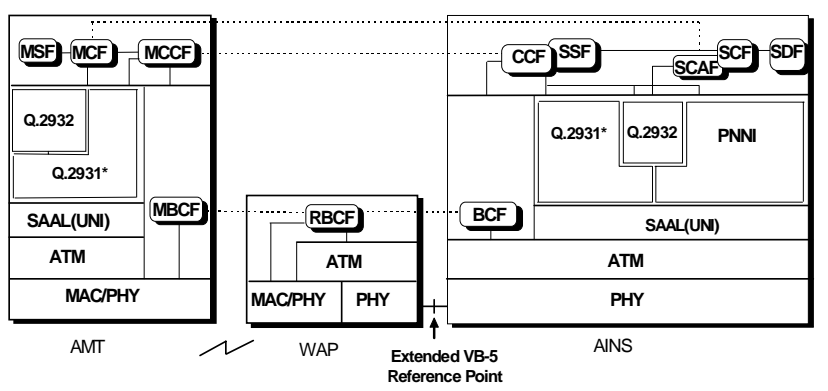

Figure 2 - Wireless Access Network Protocol Stacks

The Service Control Function (SCF) together with the Service Control Agent Function (SCAF) at the AINS and the Mobile Control Function (MCF) at the AMT stand for the support of IN user services and processing of mobilityrelated (non call-associated) services, such as user registration and location-related management. The SCAF acts as a mediator between the MCF and the SCF and screens incoming messages from the terminal side for non-call related procedures. SCF is supported by the Service Data Function (SDF), which actually comprises a database for storing IN mobility and service data. The Mobile Storage Function (MSF) comprises a small database in the AMT. The Service Switching Function (SSF) extends the logic of CCF to include the recognition of service control triggers and to interact with the SCF. The Bearer Control Function (BCF) and the Radio BCF (RBCF) serve for the resource allocation and handling of fixed and radio bearers within the access network, respectively. The Mobile BCF (MBCF) is the peer entity of the RBCF at the the AMT side.

In the WAP-AINS interface, an extended $\mathrm{VB}_{5}$ interface control protocol, [4], the so-called WAP-AINS Control Protocol (ASCP), serves for the handling of fixed bearer connections of the same call. The full set of the ASCP features and functions can be found in [4], [5]. The Private Network-to-Network Interface (PNNI) signaling, [6], is realized between the AINSs of the different W-ATM areas over the UNI S-AAL. The public UNI or the broadband public Network-to-Network Interface (NNI) with B-ISDN User Part (B-ISUP) and Message Transfer Part 3 (MTP3b) over the NNI S-AAL protocol can be assumed for interfacing the W-ATM system with the core network.
The mobility management (non-call associated) messages (e.g., location registration messages) exchanged between the mobile and service control functions are transported in a connectionless way based on the Q.2932 ConnectionLess Bearer Independent (CLBI) recommendation, [7] (use of FACILITY messages).

\section{Signaling Channel Handling}

In the signaling access architecture described above, a key problem that needs to be considered is the provision of a unique control or signaling channel between AMT and AINS in order to distinguish among different signaling sessions of the AMTs using the same WAP. Here, we consider that for each AMT all the signaling and control messages are transported via the same signaling connection at the UNI. Signaling Virtual Paths/Channels (SVP/SVC) are dynamically allocated for each AMT in the AMT-AINS logical interface using the services of the Metasignaling protocol, [8]. The same SVP/SVC pair is reserved by the AINS(BCF) for each particular AMT within the AINS area and it is notified to all WAPs during the signaling channel assignment phase using the ASCP, [5]. Note that when an AMT enters in a new W-ATM area a new SVP/SVC pair is assigned using the Metasignaling protocol.

Upon initial establishment of the AMT-AINS signaling connection, an SAAL instance is created at each port of the AINS for that particular AMT. Each time an AMT associates with a new WAP that belongs to the same WATM area, a binding procedure (which forms part of the W-ATM access network management), [9], is performed to associate the AMT, the SVPI/SVCI pair and the corresponding AINS port. Such an approach removes the need for re-spawning the signaling connections each time the terminal associates with a new WAP in the same AINS area, while provides for correctly addressing the AMT during the signaling interactions of the higher-layer signaling applications.

\section{SIGNALING AND CONTROL PROCEDURES}

This section elaborates on the signaling procedures ${ }^{1}$ and protocols between the W-ATM areas, where the AINSs are interconnected via PNNI, designed to support registration, call set-up and handover. The proposed protocols comprise an extension of the signaling protocols presented in [3] for single switch (AINS), local-area, wireless intelligent ATM Customer Premises Networks (CPNs).

\section{A. Registration}

Registration is performed when an AMT is switched-on or enters in the coverage area of a WAP's cell that belongs to a new AINS area. It is assumed that each WAP emits periodic beacons towards the AMTs in its coverage area

\footnotetext{
${ }^{1}$ A limited set of the protocol scenarios and only the successful operations are described here. The details of all the protocol cases and the procedures for exception conditions are omitted due to space limitations.
} 
identifying itself and the corresponding W-ATM area. Registration caters for the AMT authentication, to inform the network that the AMT user wants to be reachable for the services that the user is allowed to access and to update the information about the AMT location.

During registration, the $\mathrm{AMT}(\mathrm{MCF})$ reports to the AINS(SCF) its home ATM address. In case the current area is the home area of the AMT, the $\mathrm{SCF}_{\text {home }}$ checks the user subscription rights, authenticates the terminal and creates registration and location information in the database $\left(\mathrm{SDF}_{\text {home }}\right)$. A Temporary ATM Address is assigned to the AMT that uniquely identifies the terminal within the AINS area, and it is used for efficiency reasons as well as to increase confidentiality.

In case the AMT powers-up or enters in a area different from its home area as shown in Fig. 3, then registration involves the transfer of the registration data from the home area to the new area $(\mathrm{SCF} / \mathrm{SDF})_{\text {visited }}$, the update of the routing data in $\mathrm{SDF}_{\text {home }}$ and the deletion of the registration data from the old area, (if it is different from the home area). This approach complies with the two-tier architecture, [10], for location management. The $\mathrm{SCF}_{\text {home }}$ coordinates these actions to ensure that user data is up-todate in all the domains concerned. The above procedures are performed based on the Q.2932 FACILITY messages.

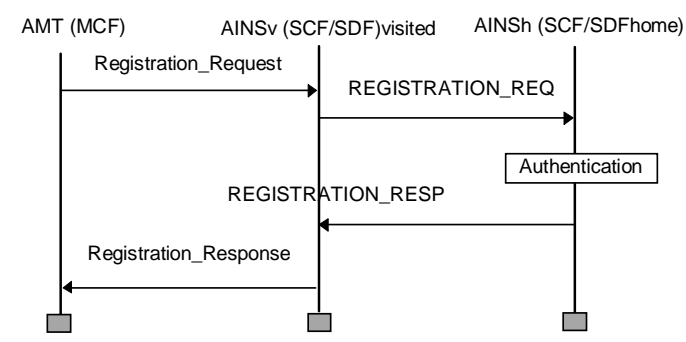

Figure 3 - AMT Registration in a Visited Area

\section{B. Call Handling}

The call control signaling interactions between the MCCF and the CCF is consistent with the Q.2931 UNI signaling, [2], as shown in Fig. 4.

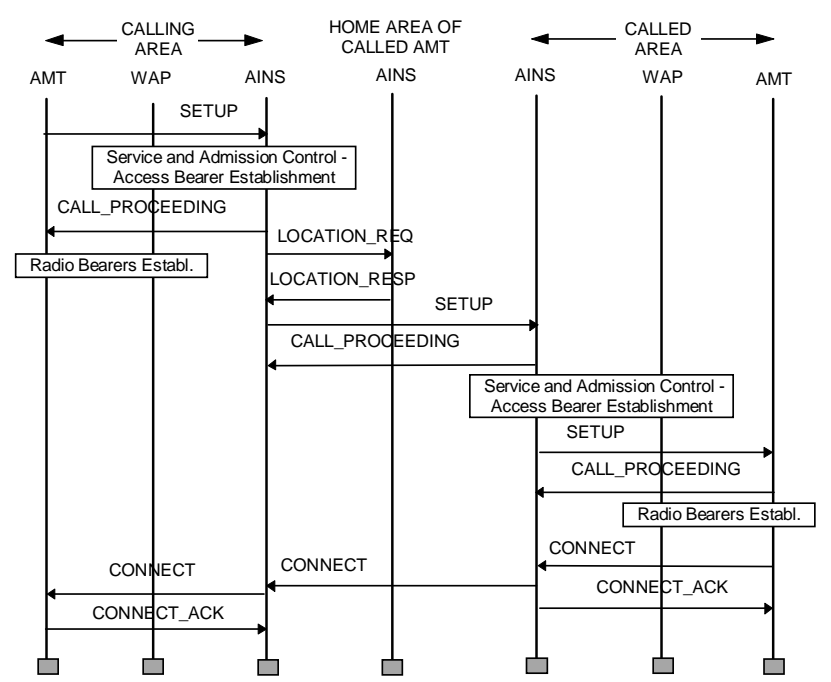

Figure 4 - Call Set-up (AMT-to-AMT call)
The CCF upon receipt of the SETUP request triggers a service check procedure towards SCF via SSF to determine if the calling user has subscribed for the requested service and checks if the user is allowed to establish bearer(s) with the requested bandwidth, [3]. The service check procedures also consider the integration of supplementary services like, e.g., conditional call forwarding, or voice mailboxes. The CCF draws an initial call acceptance decision based on the user service profile data and on the QoS requirements set by the AMT. The connection acceptance decision for the fixed and wireless part of the access network is performed by the BCF and RBCF, respectively. The task of the communication between them as well as the bearer channel establishment in the fixed access network part is realized by the ASCP, [3]-[5].

Following successful call/connection acceptance decision, the CCF then examines the dialed number of the called user to determine whether the destination is a fixed or a mobile terminal. In case the called user is an AMT in another area, the CCF contacts the SCF for the exact location of the called AMT (Fig. 4). SCF obtains the node address of the AINS of the terminating domain from the home domain $\left(\mathrm{SCF} / \mathrm{SDF}_{\text {home }}\right)$ of the called AMT. The CCF sets-up a route towards the called terminal (mobile or fixed) using standard PNNI procedures, [6]. Service check, call set-up and bearer establishment procedures for the called AMTs are similar to the those described above and will not be presented. Finally, the outgoing calls that need to be routed via the core B-ISDN are established using public UNI/NNI CC procedures.

\section{Handover Signaling and Control}

As AMTs are allowed to move freely among cells, the problem of handover within the same AINS area (intraAINS) or that of roaming between different areas (interAINS) arises. The intra-AINS handover has been described in [3]. Here, we present only the inter-AINS handovers between two WAPs of different AINS areas.

We consider that the AMT(MBCF) monitors the quality of the radio link for the current and the candidate WAPs, and decides to initiate a handover to the WAP, from which the stronger signal power is received. From the signaling viewpoint, two types of handover are identified: backward and forward. The backward, inter-AINS handover scenario is depicted in Fig. 5. 


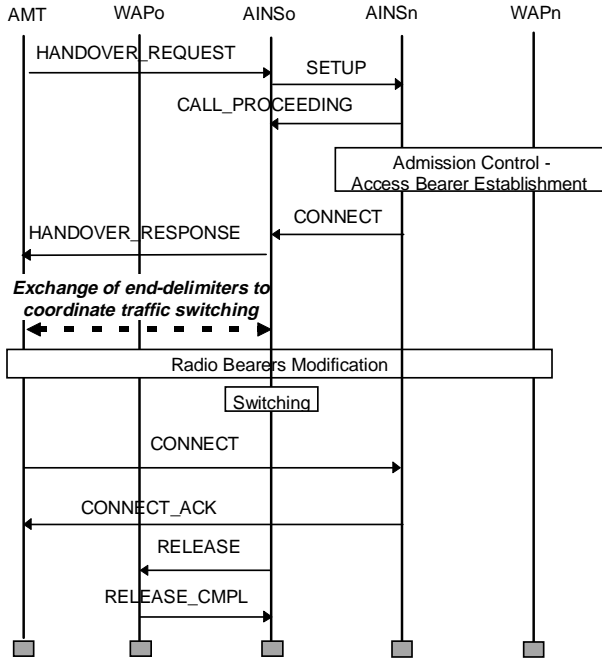

Figure 5 - Inter-AINS Handover

In case a handover needs to be performed, the AMT(MCCF) sends a HANDOVER_REQUEST message to the current AINS(CCF) denoted as AINSo transparently via the old WAP (WAPo). This message contains the AMT address, the call ID, and the target WAP (WAPn) address. The AMT may indicate the priorities of the different connections in case the candidate WAP cannot accommodate all the AMT connections in case of a multimedia call. The PNNI signaling protocol and the ASCP are also employed here for the establishment of the new bearers in the fixed network. In order to use the standard PNNI signaling Information Elements (IEs) in case of handover, we employ a reserved called party number to identify an inter-AINS handover and to distinguish it from the standard call set-up at the new AINSn(CCFn). The PNNI called party subaddress IE is used in this case to identify the WAPn that the AMT intents to move to.

When the AINSo(CCFo) is notified about the creation of the new path (receipt of the PNNI CONNECT message), issues a HANDOVER_RESPONSE message to the AMT to perform the handover. AMT(MBCF) releases its radio connection with the old WAPo(RBCF) and establishes a radio link with the new $\mathrm{WAPn}(\mathrm{RBCF})$, retrieving in this way its connectivity with the fixed network. Special ATM (and lower) layer inband signaling cell relay functions (use of end-delimiter cells) take place at AMT and AINSo to coordinate the switching of traffic and to guarantee the transport of user data at an agreed QoS level in terms of cell loss, ordering and delay. In addition, the AINSo drives the release procedures to the old WAP to notify it that the connection no longer exists and to deallocate the corresponding radio resources. Finally, the user profile migrates (not shown in the figure), as previously described in Section (3.A).

The forward handover scenario appears similar to the backward one. The main difference is that in this case the AMT communicates directly with the new WAP, so that all the handover handling messages are sent to the AINS via the WAPn and the AINSn towards AINSo performed based on the Q.2932 FACILITY messages. The latter coordinates the new path creation and the handover execution as described above.

\section{SIGNALING PERFORMANCE EVALUATION}

\section{A. Performance Model Description}

The objective of the signaling performance of the W-ATM system is to obtain measures for the mean call set-up and handover mean signaling processing delay and buffering and to study the behavior and the capacity of the access system under different signaling loads in order to reveal the maximum number of AMTs the system can accommodate in each case.

In the simulation study we have considered four W-ATM areas interconnected in a full mesh topology providing continuous coverage (see Fig. 1). Each area consists of 500 WAPs attached in one AINS. Each signaling protocol entity and each component of the IN-based functional architecture is modeled as a multiclass processing queuing system, with different service times for each message type, [11]. Under the assumption of assigning one processor per signaling component, the processing times for each signaling module and message were defined using as input the values of [3], [12], [13]. Propagation delays and cell switching times are considered of $\bullet$ sec/nsec order, and as such they have been ignored. The inter-message delay of successive user or server generated messages is assumed to be $10 \mathrm{~ms}$. Signaling links are assumed $1.5 \mathrm{Mb} / \mathrm{s}$ resulting in insignificant link emission and queuing times.

The input parameters used in the evaluation are shown in Table I. The system is tested by gradually increasing the number of AMTs per WAP and consequently the total number of AMTs under the same AINS area, assuming two different mobility scenarios. It is assumed that the moving AMTs are uniformly spread within each W-ATM area. The simulation tool used for this performance evaluation is the OPNET 2.5A.

\begin{tabular}{|l|c|}
\hline Parameter & Value \\
\hline Number of AMT users (N) & variable \\
\hline$\%$ of moving users, $\mathrm{P}_{\text {mov }}$ & variable \\
\hline $\begin{array}{l}\% \text { of moving users visiting more than one } \\
\text { areas, } \mathrm{P}_{\text {roam }}=25 \% \text { x } \mathrm{P}_{\text {mov }}\end{array}$ & variable \\
\hline $\begin{array}{l}\text { Rate of call origination/delivery } \\
\text { per AMT per hr, }{ }_{\mathrm{c}} \text { (Poisson ) }\end{array}$ & 10 calls/hr \\
\hline$\%$ of outgoing calls to a fixed terminal & $12,5 \%$ \\
\hline$\%$ of outgoing calls to an AMT in remote CPN & $12,5 \%$ \\
\hline$\%$ of incoming calls & $25 \%$ \\
\hline$\%$ of local calls to an AMT in the same CPN & $50 \%$ \\
\hline$\%$ of total busy calls & $10 \%$ \\
\hline Call Duration $(\mathrm{D})($ exponentially distributed) & $3 \mathrm{~min}$ \\
\hline Rate of AMT powering-up (down) & 2 per day \\
\hline $\begin{array}{l}\text { Rate of WAP area, intra-AINS crossings per } \\
\text { moving AMT, } \mathrm{R}_{\mathrm{C}}\end{array}$ & 3.5 per min \\
\hline $\begin{array}{l}\text { Rate of WAP area, inter-AINS crossings per } \\
\text { moving AMT, } \mathrm{R}_{\mathrm{R}}\end{array}$ & 9.5 per hr \\
\hline
\end{tabular}

Table I - Input Parameters

\section{B. Numerical Results}

In the first part of the simulation results, the performance evaluation focused on three performance measures. First, 
the mean time from the start of a call SETUP message to the receipt of the CALL_PROCEEDING message at the calling AMT (for outgoing and local calls) or the AINS (for incoming calls) is measured. This time is referred to as $\mathrm{T}_{\mathrm{cp}}$. The second performance measure is the mean call establishment delay $\left(\mathrm{T}_{\text {set-up }}\right.$ ) between two AMTs (under the same or different W-ATM areas). The expiration interval of the corresponding timers for both performance measures, defined by the existing standards, [2], serves as the upper limit for the evaluation of the call set-up processing procedures. The third performance parameter is the mean time from the start of HANDOVER_REQUEST message until the receipt of HANDOVER_RESPONSE message at the AMT $\left(\mathrm{T}_{\mathrm{ho}}\right)$. This measure represents the critical time interval for the network to decide on the handover acceptance, to create the new path and to inform the AMT to proceed with the handover.

Figs 6 and 7 illustrate the mean processing times at call set-up $\left(\mathrm{T}_{\mathrm{cp}}, \mathrm{T}_{\text {set-up }}\right)$ and handover for two different mobility scenarios for both intra and inter-AINS handover cases ( $\mathrm{T}_{\mathrm{ho}}{ }^{\text {intra }}, \mathrm{T}_{\mathrm{ho}}{ }^{\text {inter }}$ ) versus the number of users within the same W-ATM area. From these results, we obtain the maximum number of users as that the system can accommodate considering different mobility scenarios.

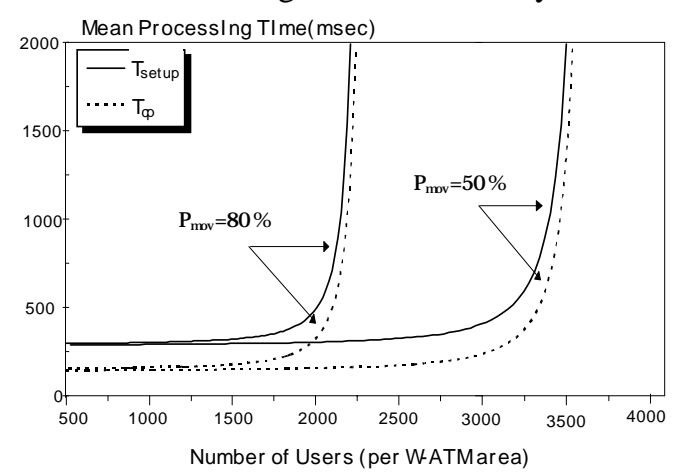

Figure 6- Mean Processing Times at Call Set-up

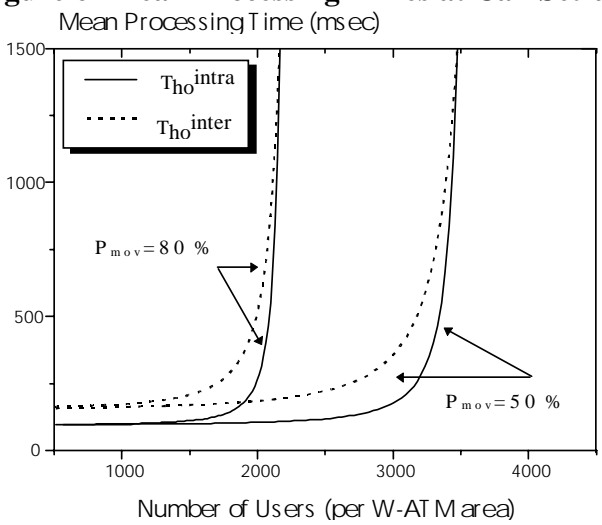

Figure 7- Mean Processing Times at Handover

As expected, as the number of AMTs increases there is a straightforward increase at the corresponding mean processing times. Note that a dramatic increase of the interested measures is observed in the high mobility scenario $\left(\mathrm{P}_{\mathrm{mov}}=80 \%\right)$. This comes from the fact that in the high mobility scenario the signaling processing entities of the system are involved many times during an AMT call's lifetime due to the high rate of handover requests that arrive. However, it has to be noticed that the presence of the MAC layer in a real wireless ATM access system will possibly increase the total signaling processing times. The second part of simulation results presents the effect of the proposed signaling architecture onto the maximum buffering requirements at the system's signaling processors. Fig. 8 illustrates the buffering requirements for the interested entities for the high mobility scenario.

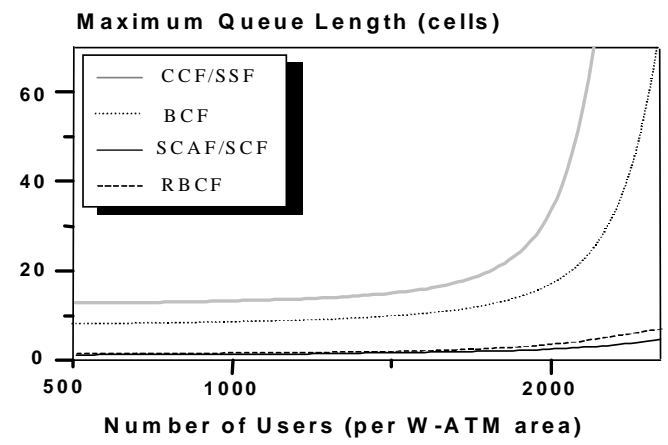

Figure 8 - System's Buffering Requirements

Under the assumption of assigning one processor per signaling component (met on existing implementations), the extracted results show that most of the signaling traffic buffering is performed within the AINS at the CCF/SSF and $\mathrm{BCF}$. The maximum buffering of signaling messages observed at the SCAF/SCF (and SDF) and RBCF queues is limited to 3-5 cells, while the maximum buffering at the $\mathrm{CCF} / \mathrm{SSF}$ and $\mathrm{BCF}$ reaches up to 70 cells. Analogous results are also observed for the low mobility scenario. From these results we can obtain that the CCF/SSF is the botlleneck in the overall performance of the system. This comes in agreement with the description of protocols in Section 3, where the CCF/SSF is highly involved in the systems's signaling operations.

\section{CONCLUSIONS}

The signaling protocol architecture considered in this paper comprises an extension of the ATM/IN signaling for application to the W-ATM scenario. Emphasis was placed on taking advantage of the well-developed broadband signaling protocol standards to allow true integration with fixed ATM. The evaluation of the proposed design captures its effect on the performance of call and mobility control and yields results, which fall within acceptable ATM signaling performance measures, and can be used for network design purposes in a large scale, private environment supporting many users.

\section{ACNOWLEDGEMENTS}

This paper is partially based on work performed by the first three authors in the framework of the RAINBOW project of the ACTS program funded by the European Commission. However, the views expressed in this paper are only those of the authors and do not represent those of the project as a whole. 


\section{REFERENCES}

[1] "RAINBOW Demonstrator Architecture and Services", $\quad$ AC015/BELL/CIT/DS/P/005/b1, RAINBOW project deliverable 01, Dec. 1995.

[2] "B-ISDN User Network Interface Layer 3 Specification for Basic Call/Bearer Control", ITU-T Rec. Q.2931, 1994.

[3] N. H. Loukas et al. "Signaling and Mobility Control for Wireless Intelligent ATM CPNs", Proc. IEEE PIMRC '98, Boston, USA, Sept 1998.

[4] "Signaling Protocols and Switching - V interfaces at the Digital Service Node (SN)", ETSI V1.5, DTR/SPS-03040, March 1996.

[5] N. H. Loukas et al. "Design of Call Control Signaling in Wireless ATM Networks", Proc. IEEE ICC '97, Montreal, Canada, June 1997.

[6] "Private Network-Network Interface Specification", ATM Forum af-pnni-0055.000, PNNI 1.0, March 1996

[7] "Generic Functional Protocol", ITU-T Draft Rec. Q.2932, 1996.

[8] "B-ISDN Metasignaling Protocol", ITU-T Rec. Q.2120, 1993.

[9] ATM Forum, "Baseline text of ATM Forum W-ATM WG", version 1.07, April 1998.

[10] B.A. Akyol and D.C. Cox, "Signaling Alternatives in a Wireless ATM Network", IEEE J. Select. Areas Commun., Vol. 15, No. 1, pp. 35-49, Jan. 1997.

[11] M. Bafutto, et al., "Capacity and Performance Analysis of Signaling Networks in Multivendor Environments", IEEE JSAC, Vol. 12, No. 3, pp. April 1994.

[12] M. Veeraraghavan et al., "A Distributed Strategy for Wireless ATM Networks", ACM Wireless Journal, Vol. 1, No. 3, pp. 323-339, Oct. 1995.

[13] I.Venieris, et. al.,"Architectural and Control Aspects of the Multi-host ATM Subscriber Loop", Journal of Network and Systems Management, Vol. 5, No. 1, pp. 55-71, Feb.-Apr. '97. 